\title{
The Comparison of Pyrazine Compounds in Non-Salted Fermented Soybean Products
}

\author{
Etsuko Sugawara, ${ }^{1}$ Tadahiko SuzUKI ${ }^{2}$ and Yoshiko YoshidA $^{3}$ \\ ${ }^{1}$ Faculty of Education, Iwate University, 3-18-33 Ueda, Morioka 020, Japan \\ ${ }^{2}$ Faculty of Agriculture, Iwate University, 3-18-8 Ueda, Morioka 020, Japan \\ ${ }^{3}$ Goshozuka 1-15-5, Miyamae-ku, Kawasaki 216, Japan
}

Received August 11, 1997; Accepted October 28, 1997

\begin{abstract}
The odor components of six different non-salted fermented soybean products in different parts of the world, Natto (Japan), Thua-nao (Thailand), Pepo (Myanmar), Libi ippa (East Bhutan), Kinema (West Bhutan) and Daddawa (Nigeria) were compared. Especially, the composition of pyrazines contributing to Natto odor was examined in these products. The odor concentrates were prepared by the method using porous polymers and were analyzed by gas chromatography and gas chromatography-mass spectrometry. Pyrazines were considered to contribute to the characteristic odor of the non-salted fermented soybean products because the compounds were detected commonly in all the products except two types of Libi ippa. The total concentration of the pyrazines was lower in Libi ippa, Kinema, and Daddawa and higher in Thua-nao and Pepo when compared with that of Natto. The composition of the pyrazines depended on the products.
\end{abstract}

Keywords: non-salted fermented soybean products, odor components, pyrazines

In our country, there are various kinds of traditional foods made from whole soybeans and soybean protein, among which Natto is one of the most interesting products because it is made from cooked soybeans and has a characteristic odor. In our previous study, pyrazines were found to be the major odor components contributing to the characteristic odor of Natto (Sugawara et al., 1985). Although pyrazines are generally thought to be generated by heating sugar and amino compounds, they were proved to form during the fermentation process of Natto by Bacillus natto (Ito et al, 1989; Sugawara et al., 1990; Yamaguchi et al., 1993). Pure cultures of $B$. natto are ordinarily selected and inoculated for fermenting Natto.

Bacillus is also used in a variety of fermented soybean products such as Nigerian Daddawa, Myanmar Pepo, Thailand Thua-nao, and West Bhutan Kinema. Because the traditional fermentation methods are used in these countries, specific bacteria are not usually identified in the fermentation. In most cases, however, strains of $B$. subtilis are supposed to be responsible for these fermentation processes (Yoshida, 1996).

The purpose of this study was to compare the odor components of the six non-salted fermented soybean products: Natto, Thua-nao, Pepo, Libi ippa, Kinema and Daddawa. Especially, the composition of pyrazines contributing to Natto odor was examined in these products.

\section{Materials and Methods}

Materials Three types of Natto were prepared in April and November in 1994 by Taishi Co. in Towada. Seven types of Thua-nao collected in Thailand were in the form of dried sheets. Nine types of Pepo were collected in Taunggii, the capital of Shan State in Myanmar, seven of them being dried and two of them having moisture like Natto. Five types of Pepo were collected in Pindaya, which is about two-h drive north of Taunggii, three of them being dried and two of them having moisture. Two types of Kinema were collected in West Bhutan, one being dried and another having moisture. Five types of Libiippa were collected in East Bhutan, all types having moisture. One type of Daddawa was collected in Nigeria. All of these samples differed in shape and size due to the difference in suppliers. Most of the characteristic odors in the non-salted fermented soybean products were similar to the odor of Natto. All of the samples were collected locally during March and April in 1994 and were frozen until needed for the experiments.

Tetramethylpyrazine (TMP)(Ogawa Koryo, Inc., Tokyo) was used for determining the yields of the pyrazines by the method with porous polymers.

Preparation of odor concentrates of the non-salted soybean products The non-salted soybean product $(20 \mathrm{~g})$ was added with $180 \mathrm{ml}$ of distilled water and mashed. The suspension was centrifuged at $4000 \mathrm{rpm}$ for $15 \mathrm{~min}$ at $5^{\circ} \mathrm{C}$. The supernatant was passed through the column packed with purified Tenax TA $(2.0 \mathrm{~g})$. The odor components in the supernatant absorbed by Tenax TA were desorbed slowly with purified diethyl ether $(50 \mathrm{ml})$. The ether eluents were added with the internal standard and dehydrated over anhydr. sodium sulfate, followed by distillation of the ether under atmospheric conditions. Two or three replicates were made for preparing odor concentrates of each sample.

Gas chromatography (GC) and gas chromatography- 
mass spectrometry (GC-MS) analysis GC analysis was carried out using a Shimadzu GC-14A gas chromatograph equipped with FID and FPD. The column was a $0.25 \mathrm{~mm} \times$ $30 \mathrm{~m}$ DB-WAX fused silica capillary column. The oven temperature was programmed from 40 to $200^{\circ} \mathrm{C}$ at the rate of $3^{\circ} \mathrm{C} / \mathrm{min}$. The temperature of the injection port was maintained at $210^{\circ} \mathrm{C}$. The flow rate of carrier gas $(\mathrm{He})$ was $0.8 \mathrm{ml} /$ min. The peak areas were calculated using a Shimadzu Chromatopack C-R4A integrator. The concentrations of odor components were calculated based on the ratio to peak areas of the internal standard. GC-MS analysis was carried out using a Hewlett Packerd 5972 Series mass spectrometer combined with a Hewlett Packerd 5890 Series II gas chromatograph equipped with a $0.25 \mathrm{~mm} \times 30 \mathrm{~m}$ DB-WAX fused silica capillary column. The oven temperature was programmed from 40 to $200^{\circ} \mathrm{C}$ at the rate of $3^{\circ} \mathrm{C} / \mathrm{min}$. The ionizing energy was $70 \mathrm{eV}$. Most peaks were identified by comparison of their mass spectra and retention data on GC with those of authentic compounds.

The recovery of TMP TMP (approx. $0.002 \mathrm{~g}$ ) was dissolved in $200 \mathrm{ml}$ of distilled water to obtain a solution of approx. $10 \mathrm{ppm}$ TMP. The solution was passed through the column packed with purified Tenax TA $(2.0 \mathrm{~g})$. The concentrate of TMP was prepared and analyzed by GC in the same way as the odor concentrates of the non-salted soybean products.

\section{Results and Discussion}

Comparison of pyrazine compound composition in the non-salted fermented soybean products Pyrazines, which are known to contribute to the characteristic odor of Natto, were also likely to be common in the other non-salted fermented soybean products and to contribute to their characteristic odor. The composition and the concentration of pyrazine compounds were compared among Natto and the other non-salted fermented soybean products. Table 1 shows the lowest, the average, and the highest concentrations of each pyrazine compound.

In the Natto odor concentrate, eight pyrazines were identified including 2,5-dimethylpyrazine as the major component. All of the types of Natto had almost the same concentration of each pyrazine compound.

Pyrazines were detected in all seven types of Thua-nao. As is shown in the Table 1, nine pyrazine compounds were identified. The composition of pyrazines depended on the samples. The pyrazine compound of the highest concentration was TMP in 4 types of Thua-nao and trimethylpyrazine in the other 3 types.

Pyrazines were detected in all 9 types of Pepo in Taunggii. Nine pyrazine compounds were identified. Two types of these

Table 1. Comparison of pyrazines in non-salted fermented soybean products. ${ }^{a}{ }^{a}$

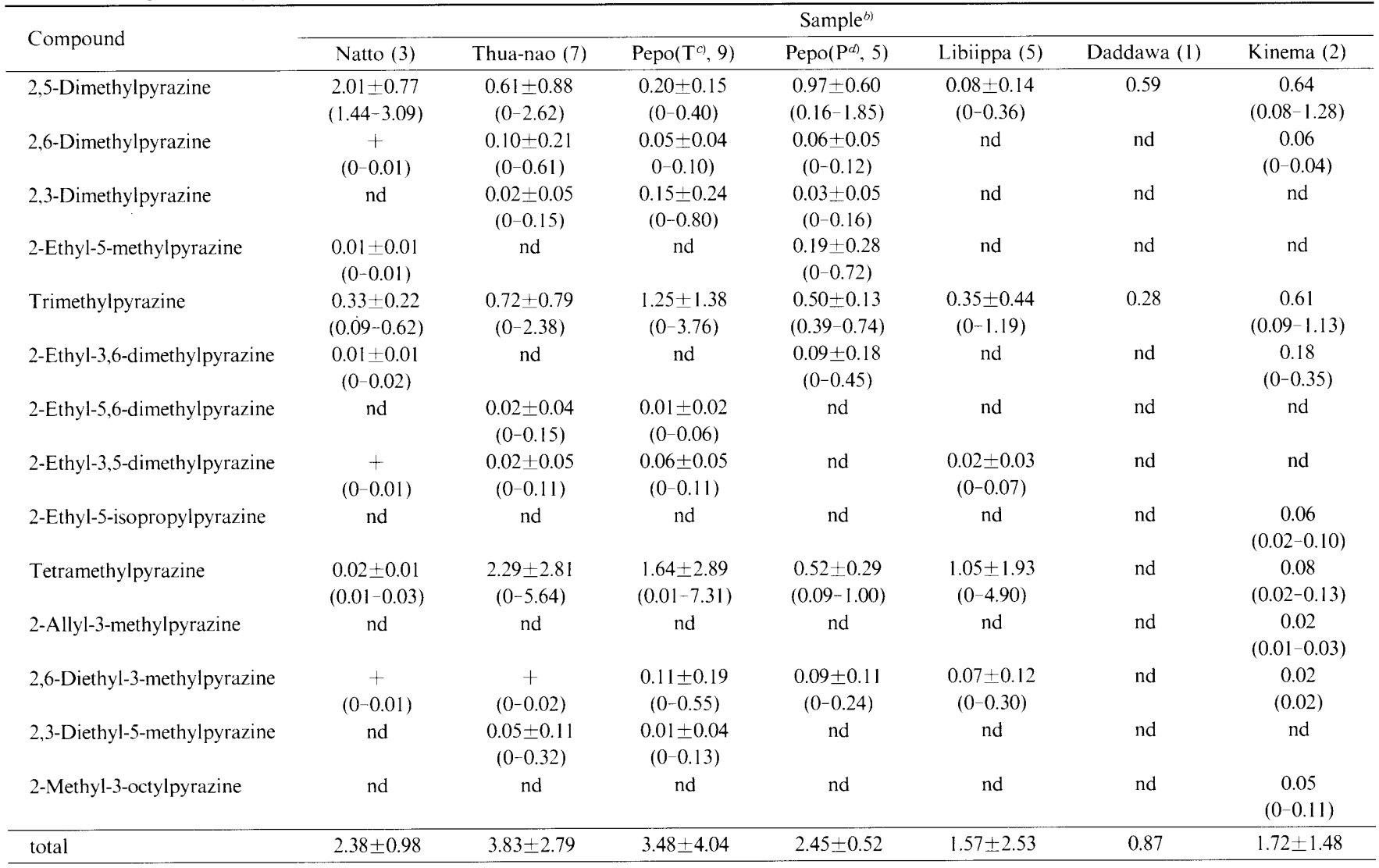

a) $\mathrm{ppm}$ : mean value $\pm \mathrm{SD}$ (minimum-maximum)

b) (sample numbers)

c) T: Taunggii

a) P: Pindaya

nd: not detected, $+:<0.01$ 
Pepo samples contained pyrazines in relatively high concentrations, with approx. $7 \mathrm{ppm}$ TMP and approx. $3 \mathrm{ppm}$ trimethylpyrazine at the same time. In 5 types of Pepo, however, the concentration of each pyrazine compound was less than $1 \mathrm{ppm}$. It shows that the composition of pyrazine compounds depended on the types of Pepo collected in Taunggii.

Pyrazines were also detected in all of 5 types of Pepo collected in Pindaya. Eight pyrazine compounds were identified. In 3 types of these Pepo samples, the pyrazine compound of the highest concentration was 2,5-dimethylpyrazine. Any pyrazine compound in the other 2 types was less than 1 ppm. The composition of pyrazines depended on the types of Pepo collected in Pindaya, too.

No pyrazine compounds were detected in 2 out of 5 types of Libi ippa. Among the other 3 types of Libi ippa having pyrazine compounds, one type had nothing but an extremely low concentration of trimethylpyrazine. It resulted in the low average concentration of pyrazines in Libi ippa. Considering that pyrazines in Natto are produced by $B$. natto, it is possible to assume that Libi ippa is fermented by either $B$. subtilis, which is similar to $B$. natto, or by totally different yeast or molds.

Two pyrazines were detected in Daddawa. Nine pyrazines were detected in both of the two types of Kinema, which contained 2,5-dimethylpyrazine and trimethylpyrazine as the major components.

Thus, pyrazines were detected in all of the non-salted fermented soybean products except 2 types of Libi ippa. The results suggest that $B$. subtilis, which is similar to $B$. natto, is involved in the fermentation process in the non-salted fermented soybean products in the same way as in Natto. Natto is fermented by pure cultures of $B$. natto under completely controlled conditions. As for the other non-salted fermented soybean products, however, the fermentation conditions including the microorganisms cannot always be the same. This may cause quite a different odor composition within the same product from different suppliers.

The recovery of TMP In order to calculate the concentration of pyrazines in the non-salted fermented soybean products, TMP was recovered by preparing the odor concentrate using porous polymers. The average yield of five replicates was $20.1 \%$.

Comparison of the total concentration of pyrazines in the non-salted fermented soybean products Figure 1 shows the total concentration of pyrazines and the composition of the major pyrazines (2,5-dimethylpyrazine, trimethylpyrazine, and TMP) in the odor concentrates in each sample. The total concentration of pyrazines was based on the yields determined from the recovery experiment for TMP.

The total concentration of pyrazines was approx. $12 \mathrm{ppm}$ in Natto and was supposedly in the range of 4 to $19 \mathrm{ppm}$ in the other non-salted fermented soybean products. Depending on the samples, the total concentration of pyrazines in the Thua-nao and Pepo in Taunggii was about 1.5 times that in Natto. The high concentration was probably caused by the dried form of Thua-nao and Pepo samples. If these samples had a water content as high as that of Natto, the total concentration of pyrazines in these products would be half ppm

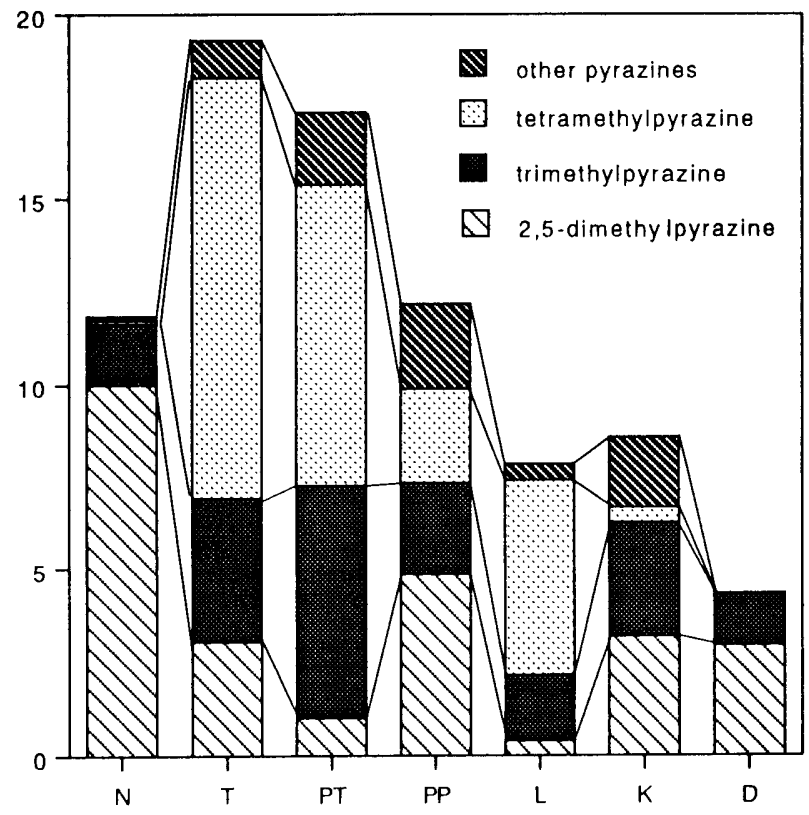

Fig. 1. The total amount of pyrazines in non-salted fermented soybean products. N: Natto, T: Thua-nao, PT: Pepo (Taunggii), PP: Pepo (Pindaya), L: Libi ippa, K: Kinema, D: Daddawa.

and would be calculated to be almost the same as that of Natto. Libi ippa, Kinema and Daddawa had lower concentrations of the total pyrazines than Natto in spite of the water content being as high as in Natto.

The composition and the mean concentration of pyrazines in Natto were similar to those of the samples from Thailand and Myanmar but differed from those of the samples in Bhutan and Nigeria. The flora of microorganisms in Thuanao and Pepo were considered to be similar to those of Natto, but those in Libi ippa, Kinema and Daddawa differ slightly from those in Natto.

High concentrations of 2,5-dimethylpyrazine, trimethylpyrazine, and TMP were detected in many of the non-salted fermented soybean products. These pyrazines have a "burnt" odor which is extensively found in roasted foods. The odor threshold value has been reported to be $0.8-1.8 \mathrm{ppm}$ for 2,5-dimethylpyrazine, 0.4-1.8 ppm for trimethylpyrazine, and 1-10 ppm for TMP (Mihara \& Masuda, 1988; Leffingwell \& Leffingwell, 1991). The concentrations of these pyrazines in the non-salted fermented soybean products were apparently higher than the threshold values above. Therefore, these pyrazines are thought to enhance the preference for the non-salted fermented soybean products.

Acknowledgments This study was supported partly by Takano Life Science Research Foundation. The field studies conducted in Myanmar and Bhutan were funded by the Ajinomoto Foundation for Dietary Culture.

\section{References}

Ito, T., Sugawara, E., Myanohara, J.,Sakurai, Y. and Odagiri, S.(1989). Effect of amino acids as nitrogen sources on microbiological formation of pyrazines. J. Jpn. Soc. Food Sci. Technol, 36, 762-764. 
Leffingwell, J.C. and Leffingwell, D. (1991). GRAS flavor chemicalsdetection thresholds. Perfum. Flavor., 16, 2-19.

Mihara, S. and Masuda, H.(1988). Structure-odor relationships for disubstituted pyrazines. J. Agric. Food Chem., 36, 1242-1247.

Sugawara, E., Ito, T., Odagiri, S., Kubota, K. and Kobayashi, A. (1985). Comparison of compositions of Natto and cooked soybeans. Agric. Biol. Chem., 49, 311-317.

Sugawara. E., Ito, T., Yonekura, Y., Sakurai, Y. and Odagiri, S. (1990). Effect of amino acids on microbiological pyrazines forma- tion by $B$. natto in chemically defined liquid medium. Nippon Shokuhin Kogyo Gakkaishi, 37, 520-523 (in Japanese).

Yamaguti, N., Toda, T., Teramoto, T., Okuhira, T., Sugawara. E. and Ito, T. (1993). Effect of sugars on microbiological pyrazine formation by Bacillus natto in synthetic liquid medium. Nippon Shokuhin Kogyo Gakkaishi, 40, 841-848 (in Japanese).

Yoshida, Y. (1996). Natto: Non-salted soybean fermentation in tropical Asia. In "Ethnobiology in Human Welfare," ed. by S.K. Jain. Deep Publications, New Delhi, pp. 13-19. 